\title{
Material multimedia para la integración de contenidos en asignaturas y grados del Área de la Salud: Transporte de membrana.
}

Eva Serna $^{a}$, Teresa San-Miguel ${ }^{b}$, Javier Megías ${ }^{\mathbf{b}}$, María Dolores Mauricio ${ }^{\mathbf{a}}$

aDepartamento de Fisiología, 8 QIVHUWbاGHMDaQFD.Eva.serna@uv.es, m.dolores.mauricio@uv.es

bepartamento de Patología, Universitat de Valencia. Teresa.Miguel@uv.es, Javier.megias@uv.es

\section{\$EWWDW}

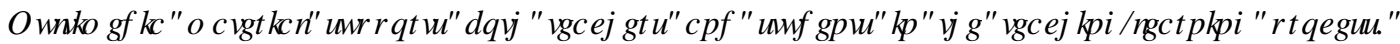

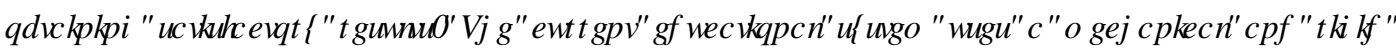

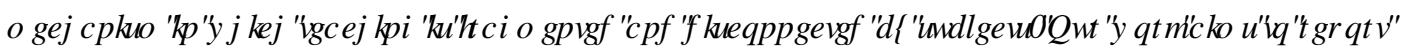

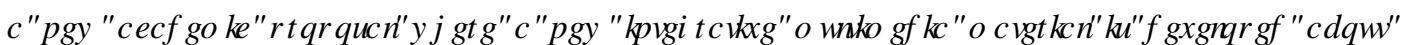

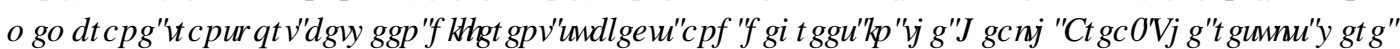
YHUपИUMDFWRU

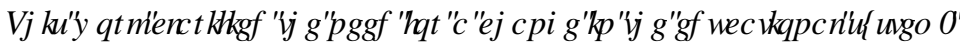

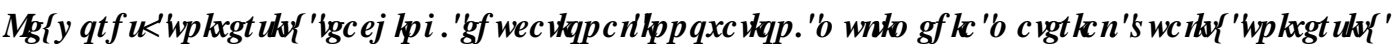 WDFKIQ $\square$ P XOMGDFLSQQDU IIQWUUWRQ}

\section{HXP HQ}

]

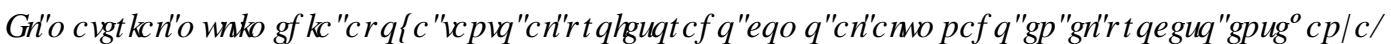

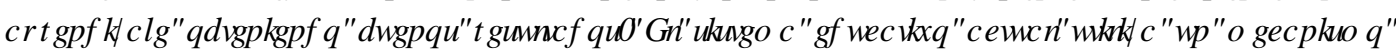

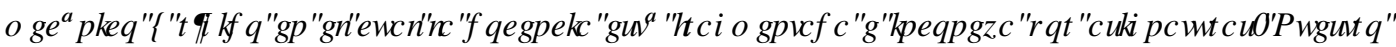

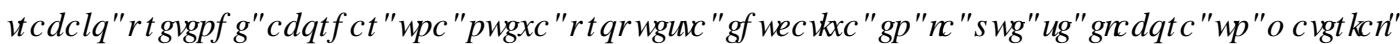

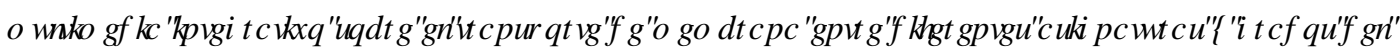

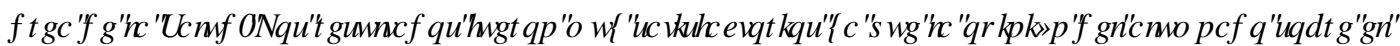

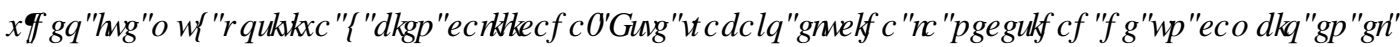
UWHP DHCXFDUARII

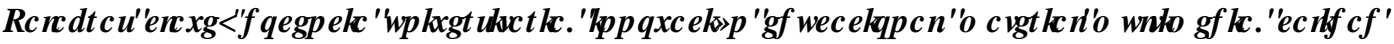

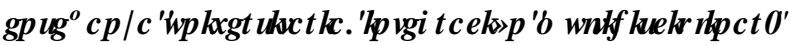

\section{Introducción}

La docencia universitaria necesita de nuevas TICs o medios para transmitir y presentar la información. Los materiales multimedia presentan la información con imágenes, sonidos y texto simultáneamente y son una gran alternativa, como muchos autores han destacado, para impartir una docencia de calidad (Marquès, 2000; Guerra-Ojeda et al., 2016; Mauricio et al., 2017). En nuestro equipo de investigación docente hemos observado que el hecho de introducir materiales de elaboración propia para explicar y reforzar algunas 
partes del temario ofrece buenos resultados tanto a nivel académico como en cuanto al incremento de la motivación de los alumnos por la asignatura, uno de los principales motores del aprendizaje (Serna et al., 2018).

Para la elaboración de estos recursos se necesita un profesorado que esté formado para que sea capaz de crear su propio material multimedia. Para ello, la Universitat de València convoca proyectos de innovación docente que, además, en una de sus modalidades ofrecen la ayuda técnica para elaborar dicho material. Nuestro equipo docente desarrolló un proyecto concedido en la convocatoria durante el curso académico 2019-2020 (Projectes innovació Código proyecto: UV-SFPIE_PID19-1095842).

Este tipo de recurso multimedia apoyaría tanto al profesorado como al alumnado en el proceso enseñanzaaprendizaje obteniendo mejores resultados. Nos basamos en la idea de que "entender mediante conceptos y entender a través de la vista se combinan en una suma positiva reforzándose o al menos integrándose el uno en el otro" (Sartori, 1998), lo que viene a fortalecer la opinión de que la cultura escrita y audiovisual juntas dan lugar a un mayor éxito. Riera señala que los materiales educativos multimedia no pretenden solamente posibilitar un aprendizaje memorístico y conceptual, sino posibilitar un tipo de aprendizaje comprensivo y constructivo, adaptado a las necesidades, a los intereses y al propio ritmo de aprendizaje de los estudiantes (Riera, 2000).

Por otra parte, el sistema educativo en todas las etapas está planteado por asignaturas impartidas de manera inconexa y fragmentada mediante un modelo mecánico y rígido. Donde, además, los docentes no suelen interactuar entre las diferentes áreas y asignaturas para poder elaborar un destacado material integrador y completo para el alumnado.

Edgar Morin propone un modelo educativo en el que -ningún objeto o acontecimiento (que deba ser analizado por la ciencia) se encuentra aislado o desvinculado, sino que éste aparece dentro de un sistema complejo, desde donde entabla una gama de relaciones con otros objetos, ya sea "internos" (con otros objetos componentes del sistema), o "externos" (con elementos del ambiente, del entorno o del contexto de ese sistema). A grandes rasgos, esta propuesta intenta integrar el enfoque global y complejo del mundo con una educación que tome en cuenta esta visión. Así, todo conocimiento humano que nos permita tomar conciencia de que somos tan sólo una parte de un sistema más general (complejo y en constante interacción), será bienvenido para formar parte de la educación. Por ello, desde esta perspectiva se habla, no sólo de una nueva educación, sino de una "reforma del pensamiento" (Morín, E, 2004; Pereira Chaves, J.M, 2010).

Con estos cimientos filosóficos nos adentramos en nuestro escenario del Área de la Salud, donde en un pequeño mundo coexisten dos asignaturas la Fisiología y la Biología Celular. En la actualidad, estas dos disciplinas se imparten de manera independiente y, esta situación, no permite una buena integración de contenidos de manera sencilla para el alumnado. Una educación que integre todos los niveles puede ser el medio más adecuado y efectivo para el desarrollo de las capacidades del alumnado. Esto le permitirá comprender el conocimiento humano más complejo desde un punto de vista amplio e integrador y cuyo resultado final en su aprendidaje será más que la suma de las partes de manera individual.

\section{Objetivos}

El presente trabajo tiene como objetivo desarrollar una propuesta educativa diferente donde docentes de ambas asignaturas se reúnan y confeccionen un material multimedia que explique el transporte de membrana integrando y unificando contenidos, tanto desde el punto de vista de la funcionalidad como la de sus elementos celulares. Posteriormente, los objetos de aprendizaje creados se compartirán con el 
alumnado mediante TICs institucionales, concretamente en "Aula Virtual" y en el blog "Biopato" (http://biopato.blogs.uv.es).

De esta manera, se busca incrementar el nivel de conocimiento en el alumnado y compartir la experiencia de creación y ejecución de un proyecto transversal e integrador entre docentes de diferentes departamentos en un momento de transformación académica basándonos en lo que rige el espacio europeo de enseñanza superior. Además, la utilización de material multimedia en docencia ayuda a los alumnos a integrar las TIC en sus procesos de aprendizaje y la utilización de plataformas propias de la UV pone en valor la apuesta de nuestra institución por estas herramientas.

Es por ello que podemos resumir los objetivos de este trabajo en los siguientes subobjetivos:

Objetivos específicos:

1. Confeccionar un material multimedia integrador.

2. Analizar la opinión del alumnado mediante una encuesta anónima y voluntaria sobre el material multimedia proporcionado.

\section{Desarrollo de la innovación}

Durante el curso 2019-2020 se elaboró un material multimedia mediante una buena coordinación docente interdepartamental que permitiría al alumnado integrar sus conocimientos sobre el transporte de membrana tanto desde el punto de vista de la Fisiología como de la Biología Celular. Este material se publicó en el siguiente enlace: https://youtu.be/6I2dwCcHrVg y su duración es de aproximadamente $8 \mathrm{~min}$.

Durante el curso 2020-2021 se puso a disposición del alumnado de ambas asignaturas. Concretamente, la experiencia se llevó a cabo con el alumnado matriculado en:

Fisiología I (Código 34070) del Grado en Farmacia: asignatura de $2^{\circ}$ curso de 6 ECTS, troncal con 50 matriculados en el grupo $\mathrm{D}$, de los cuales 24 participaron en el estudio.

Fisiología humana (Código 33002) del Grado en Fisioterapia: asignatura de $1^{\text {er }}$ curso de 9 ECTS, troncal con 51 matriculados en el grupo B, de los cuales 23 participaron en el estudio..

Biología (Código 34442) del Grado en Medicina: asignatura de $1^{\text {er }}$ curso de 6 ECTS, formación básica con 84 matriculados en el grupo B y 79 en el grupo V, de los cuales 25 y 17 participaron en el estudio.

Este material multimedia fue realizado gracias a la concesión de un proyecto de Innovación Docente titulado "Integración de contenidos de Biología Celular y Fisiología mediante TICs (I): Transporte de membrana" con Código: UV-SFPIE_PID19-1095842 y coordinado por Eva Serna García.

La encuesta anónima y voluntaria que se les proporcionó, tras la visualización del material multimedia, fue compartida utilizando Google Forms y se detalla a continuación:

(c)) BY-NC-ND 2021, Universitat Politècnica de València

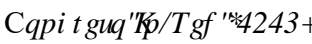




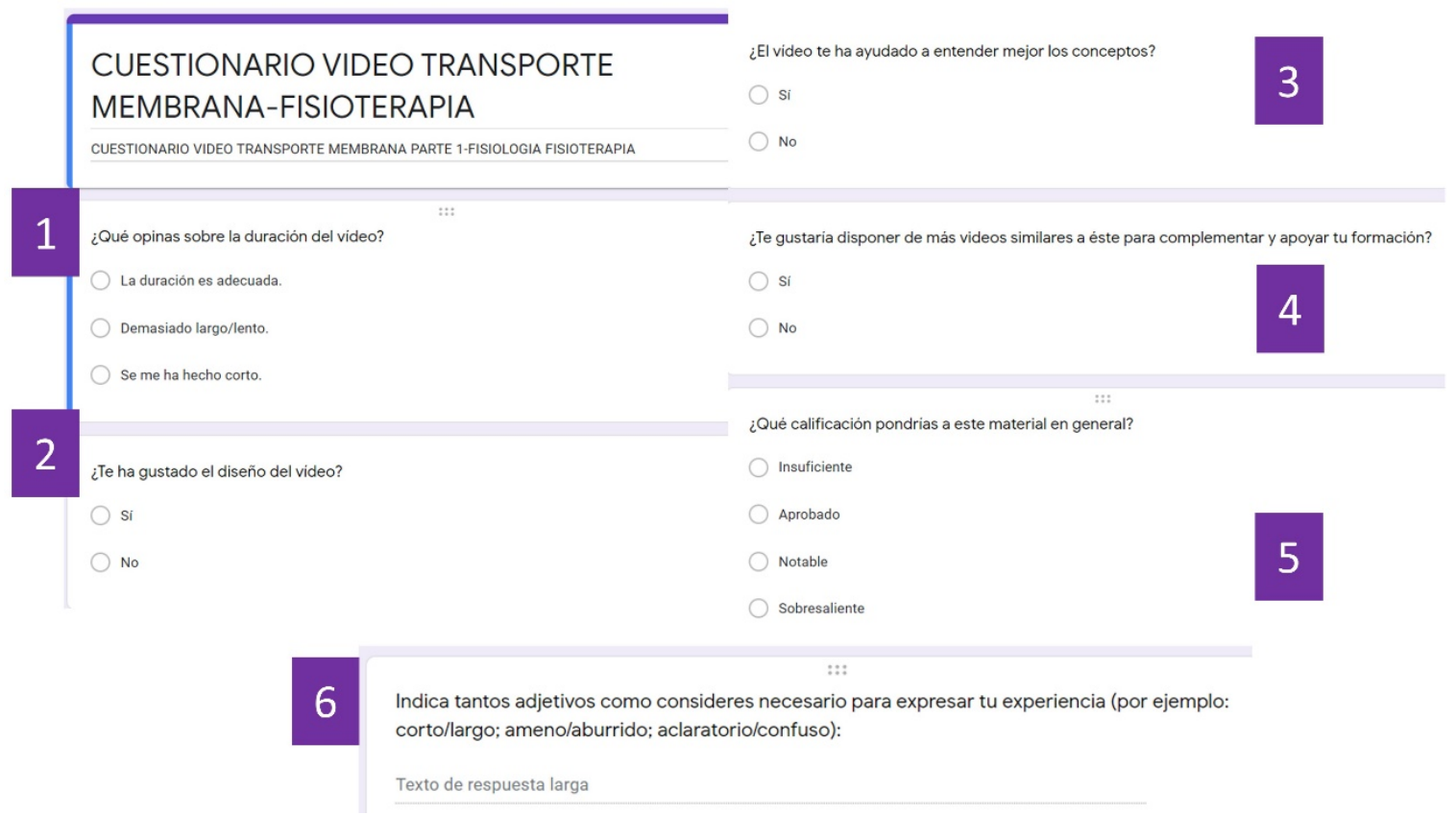

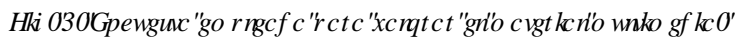

\section{Resultados}

El material multimedia se elaboró con la finalidad de profundizar en aspectos como generalidades de la membrana y transporte pasivo dentro del marco de Transporte de membrana utilizando material docente de los dos departamentos participantes: Fisiología y Patología (Biología) de la Universitat de València. El número de visualizaciones hasta la fecha en Youtube es de 2511. Y las valoraciones como "đ্NH" fueron 38, y una negativa.

La encuesta realizada al alumnado para saber la opinión de dicho material (Fig.1) nos proporcionó los siguientes resultados. Todos los participantes de todos los grados y grupos les gustó el diseño del vídeo (100\% de un total de 89 participantes). También valoraron que les había ayudado a entender mejor los conceptos (100\% de 89 participantes) y nos animaban a elaborar más vídeos similares a éste para complementar y apoyar su formación (100\% de 89 participantes).

En cuanto a la duración del vídeo hubo más discrepancias entre grados y grupos (Fig.2). 
FISIOTERAPIA

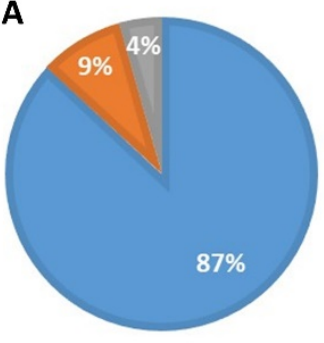

GRUPO V MEDICINA

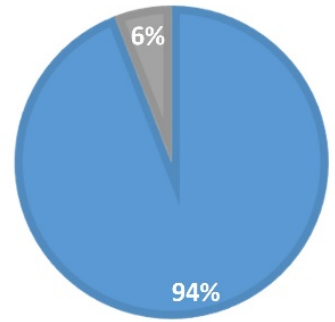

FARMACIA
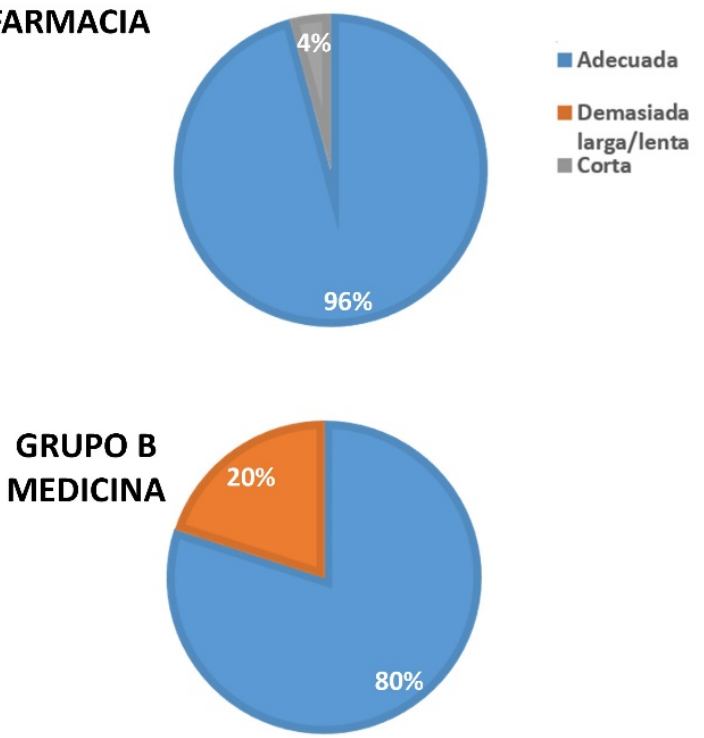

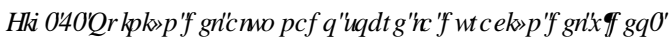

La valoración de la duración del vídeo fue positiva, considerándola mayoritariamente adecuada ( $87 \%$ en Grado en Fisioterapia, 96\% en Grado en Farmacia, 94\% en grupo V del Grado en Medicina y 80\% en el grupo B del Grado en Medicina), observamos que a un 20\% del alumnado del grupo B de Medicina les hubiera gustado un vídeo más dinámico y más corto. Este porcentaje fue menor en el Grado en Fisioterapia con un $9 \%$, mientras que en el resto de grados no se recogió esa opinión.

La calificación del material multimedia en general fue muy alta, y en todos los grados se situó entre el notable y sobresaliente (Fig. 3). No obstante, cabe destacar que el grupo B de Medicina fue el grupo más crítico a la hora de valorar y calificó el material con un mayor porcentaje de notables que de sobresalientes.

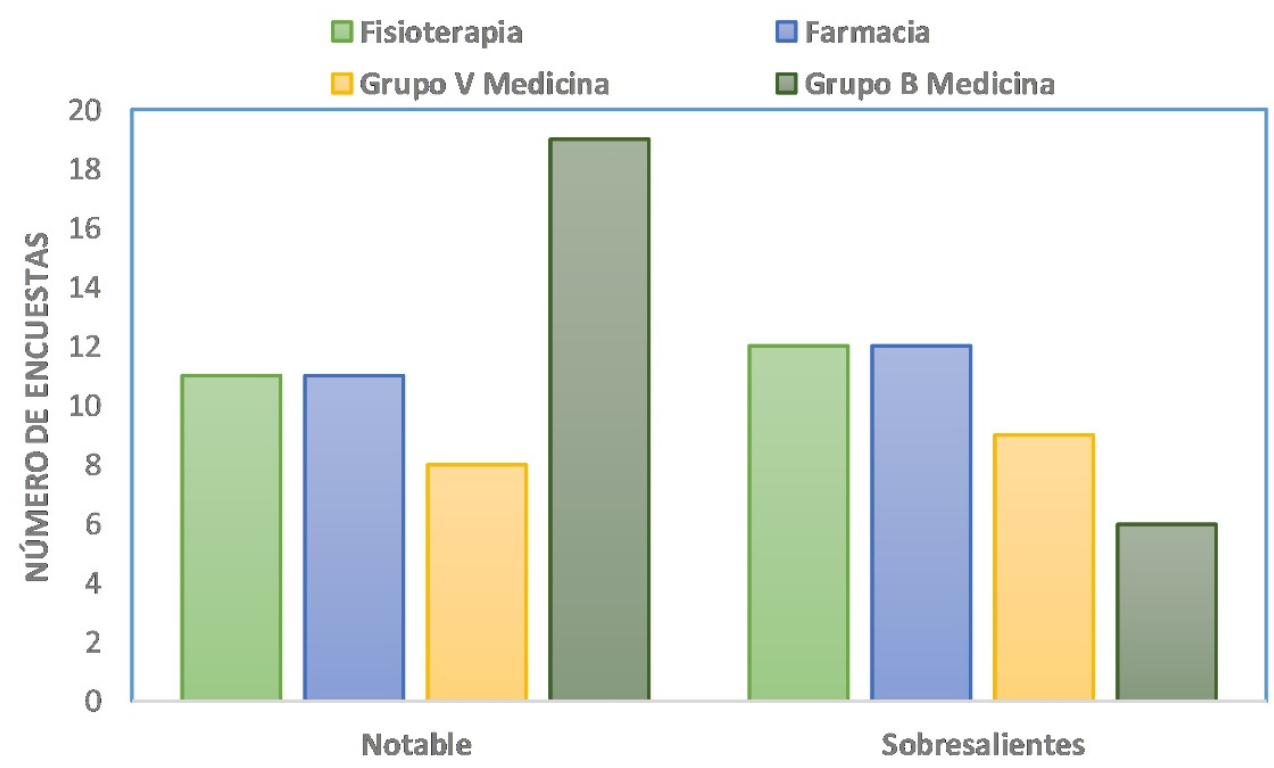

) $L J \amalg \square \& D O I F D F I y Q G H D P D U H D O P X Q M P H A D D$

(c)) BY-NC-ND 2021, Universitat Politècnica de València

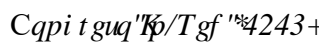




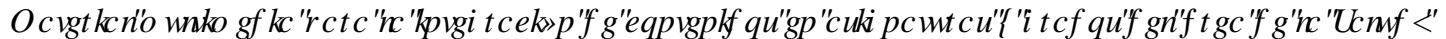 7UDQSRUKIGHP H EUQDD}

Sobre la opinión libre de los alumnos (punto 6 de la encuesta proporcionada) obtuvimos una alta participación y solo 28 estudiantes no valoraron, de los cuales 13 perteneciann al grupo B de Medicina, 10 a Farmacia, 3 al grupo V de Medicina y 2 a Fisioterapia.

Como muestra la Tabla 1 el adjetivo más empleado y significativo fue "aclaratorio", y después "ameno". Cabe destacar que ambos adjetivos tuvieron porcentajes similares de uso en los distintos grados y grupos (Fig. 4). Por tanto, es muy representativo como valoración general del vídeo. Después las palabras como "preciso", "adecuado", "claro", "útil", "interesante" y "visual” fueron las más repetidas (Tabla 1).

Otros adjetivos empleados fueron: "ayuda/refuerzo" (4), "directo" (3), "apropiado" (2), “completo" (2), “correcto" (1), "ingenioso" (1), "perfecto" (1), “accesible” (1), “dinámico" (1), "voz de locutor agradable" (1), "productivo" (1) y "esquemático" (1).

Tabla 1. Valoración libre del alumnado sobre el material multimedia, separada por grupos y grados. Los números indican las veces que se repite ese adjetivo.

\begin{tabular}{|c|c|c|c|c|c|}
\hline $\begin{array}{l}\text { Adjetivos } \\
\text { empleados }\end{array}$ & Fisioterapia & Farmacia & $\begin{array}{l}\text { Medicina } \\
\text { Grupo V }\end{array}$ & $\begin{array}{l}\text { Medicina } \\
\text { Grupo B }\end{array}$ & TOTAL \\
\hline Aclaratorio & 15 & 10 & 10 & 11 & 46 \\
\hline Ameno & 7 & 7 & 6 & 8 & 28 \\
\hline Preciso & 3 & 1 & 3 & 3 & 10 \\
\hline Adecuado & 8 & 0 & 0 & 1 & 9 \\
\hline Claro & 5 & 2 & 1 & 1 & 9 \\
\hline Útil & 7 & 0 & 0 & 1 & 8 \\
\hline Interesante & 3 & 2 & 1 & 1 & 7 \\
\hline Visual & 1 & 2 & 2 & 1 & 6 \\
\hline
\end{tabular}




\section{ACLARATORIO}

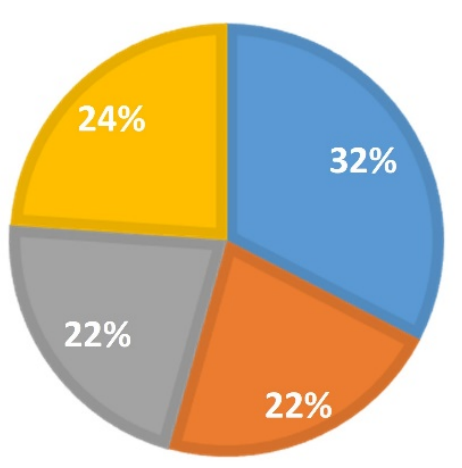

- Fisioterapia

- Farmacia

Grupo V

Medicina

Grupo B

Medicina

\section{AMENO}

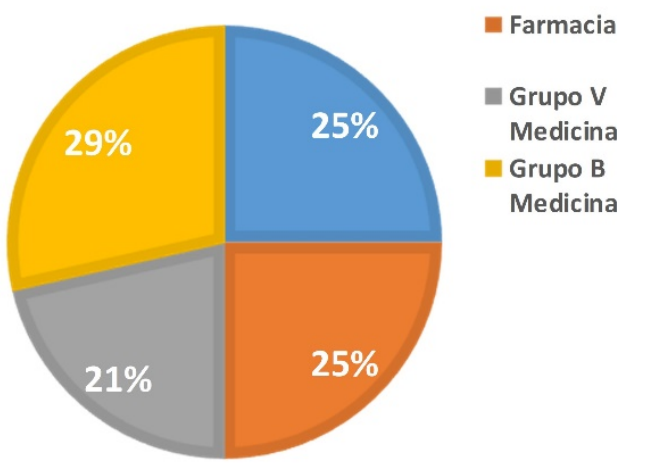

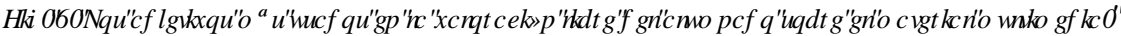

\section{Conclusiones}

El presente proyecto desarrolló una propuesta educativa donde docentes de ambas asignaturas se reunieron y confeccionaron un material multimedia explicando el transporte de membrana integrando y unificando contenidos, tanto desde el punto de vista de la funcionalidad (Fisiología) como la de sus elementos celulares (Biología Celular). De esta manera, se pretende facilitar la asimilación de los contenidos por el alumnado y compartir la experiencia de creación y ejecución de un proyecto transversal, que integra a varios docentes de diferentes departamentos con preocupaciones comunes y motivación para implementar las ideas que se proponen en el espacio europeo de enseñanza superior. La experiencia del profesorado fue muy enriquecedora y nos abre una ventana al futuro muy alentadora gracias a la buena opinión de nuestro alumnado.

En líneas generales, la duración del vídeo fue adecuada para todos los grados y grupos estudiados. Todos los participantes opinaban que les ayudaba mejor a entender los conceptos, y que les gustaría disponer de vídeos similares en su formación. El diseño les gustó y lo consideraron significativamente un material aclaratorio y ameno.

La calificación del vídeo fue buena pero no excelente para todos los partipantes, destacando el grupo B del Grado en Medicina como el más crítico a la hora de valorar. Esto nos lleva a pensar, como en muchas ocasiones nos hemos planteado, que no para todos los grados, ni como hemos observado con este trabajo, para todos los grupos, existe un material perfecto ya que el perfil del alumnado y su ambiente universitario es muy heterogéneo. Es por ello, que para futuros trabajos se debería tener en cuenta todos estos datos recogidos y corroborarlos con nuevos estudios.

Como conclusión final podemos afirmar que el material multimedia es una herramienta que se debería usar en la docencia universitaria para integrar contenidos entre asignaturas, ya que permite adquirir un conocimiento más amplio y conectado. Concretamente en nuestro ámbito es un error estudiar el organismo de manera fragmentada y no como un todo. Este trabajo elucida la necesidad de un cambio en el sistema 
educativo. Además, el hecho de realizar encuestas de opinión al alumnado sobre los materiales que elaboramos nos ayuda a los docentes a encaminarnos hacia ese cambio de una manera más segura y efectiva.

\section{Referencias}

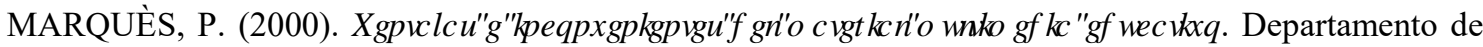
Pedagogía Aplicada, Facultad de Educación, $\mathrm{UAB}<\mathrm{http}$ ://peremarques.net/ventajas.htm $>$ [Consulta: 20 de marzo de 2018].

GUERRA-OJEDA S, SERNA E, VALLÉS SL, ALDASORO M, VILA JM AND MAURICIO MD (2016). "Multimedia: A useful tool for improving the learning of Physiology". ; ; ; 9, , , $\$ \& 21 * 5$ ( 62 " ( $\square \$ \square(\&) \square$

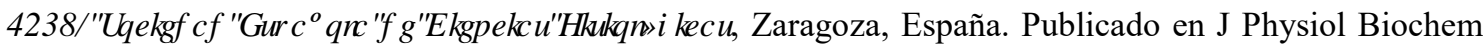
(2016) 72 (Suppl 1):S1-S111. P1-05.

MAURICIO MD, VALLÉS SL, VILA JM, ALDASORO M Y SERNA E. (2017)."Material multimedia

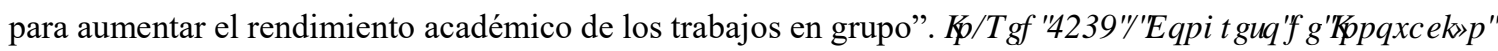

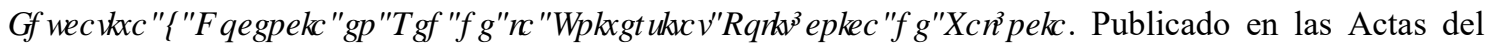
Congreso. Editorial Universitat Politècnica de València. Doi: http://dx.doi.org/10.4995/INRED2017.2017.6846

SERNA E, MEGÍAS J, OLASO G, MAURICIO MD Y SAN-MIGUEL T. (2018). "Material multimedia

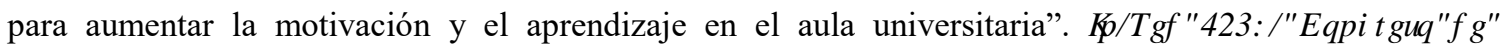

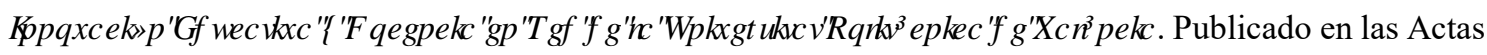
del Congreso. Editorial Universitat Politècnica de València. Doi: http://dx.doi.org/10.4995/INRED2018.2018.8608

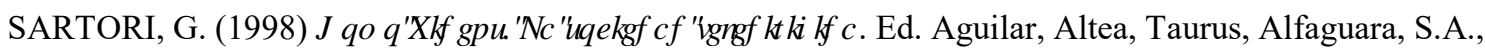
México.

RIERA, B. (2000). 3LRFHR GH GMHR $\square G H$ PDUHDOMV HGXFDUYRV PXONPHD. Recuperado de http://www.pedagogia.fcep.urv.es/educanet/recursos/edfisica/ticenedfisica/documentos/proceso_diseno.p $\mathrm{df}$

MORÍN, E. (2004). ,QURGXFFIy QDOSHQMP IHQURIFRP SGNK. México, D. F.: Editorial Gedisa.

PEREIRA CHAVES, J.M (2010). \&RQMGHDFIRQHEE UFDVGHOSHQMP IHQMRIRP S GNAICH( GJDUO RUQIHQ DIHCXFDFlyQ Revista Electrónic@ Educare Vol. XIV, № 1, [67-75], ISSN: 1409-42-58, Enero-Junio 2010. 